\title{
Nano for the Public: An Exploranation Perspective
}

Gunnar Höst, Karljohan Lundin Palmerius and Konrad Schönborn

The self-archived postprint version of this journal article is available at Linköping University Institutional Repository (DiVA):

http://urn.kb.se/resolve?urn=urn:nbn:se:liu:diva-164020

N.B.: When citing this work, cite the original publication.

Höst, G., Lundin Palmerius, K., Schönborn, K., (2020), Nano for the Public: An Exploranation Perspective, IEEE Computer Graphics and Applications, 40(2), 32-42.

https://doi.org/10.1109/MCG.2020.2973120

Original publication available at:

https://doi.org/10.1109/MCG.2020.2973120

Copyright: Institute of Electrical and Electronics Engineers (IEEE)

http://www.ieee.org/index.html

(C)2020 IEEE. Personal use of this material is permitted. However, permission to reprint/republish this material for advertising or promotional purposes or for creating new collective works for resale or redistribution to servers or lists, or to reuse any copyrighted component of this work in other works must be obtained from the IEEE. 


\title{
Nano for the Public: An Exploranation Perspective
}

\author{
Gunnar Höst, Karljohan Palmerius and Konrad Schönborn
}

\begin{abstract}
Public understanding of contemporary scientific issues is critical for the future of society. Public spaces, such as science centers, can impact the communication of science by providing active knowledge-building experiences of scientific phenomena. In contributing to this vision, we have previously developed an interactive visualization as part of a public exhibition about nano. We reflect on how the immersive design and features of the exhibit contribute as a tool for science communication in light of the emerging paradigm of exploranation, and offer some forward-looking perspectives about what this notion has to offer the domain.
\end{abstract}

Index Terms - N.2 E-learning tools $<$ N. Learning Technologies; N.6 Devices for learning $<$ N. Learning Technologies; H.5.1.b Artificial, augmented, and virtual realities $<$ H.5.1 Multimedia Information Systems $<$ H.5 Information Interfaces and Representation $(\mathrm{HCl})<\mathrm{H}$ Information Technology and Systems; N.1.e Educational simulations $<\mathrm{N} .1$ Learning environments $<\mathrm{N}$. Learning Technologies; I.6.3 Applications < I.6 Simulation, Modeling, and Visualization < I Computing Methodologies;

\section{INTRODUCTION}

$S_{d}$ CIENTIFIC discovery is intertwined with technological development, which poses both potential risks and benefits for society. The ability to critically gauge the societal implications of new scientific innovations is a crucial component of scientific literacy [1]. In this respect, nanoscience and nanotechnology (nano) is a domain of scientific endeavour associated with narratives of hope as well as fear. The significant impact that nano stands to make on humanity requires increased efforts for supporting public understanding of this area, with science centers seen as one central avenue for doing so [2]. In contributing to this mandate, we have previously developed an interactive simulation of otherwise imperceptible nanoscopic properties and interactions as part of an exhibition about nano [3]. The system, NanoSim, was designed to provide visitors with an interactive, immersive experience of the nanoworld that conveyed scientific concepts related to potential risks and benefits of nanotechnology [4].

Based on the observation that rapid developments in visualization and interaction technology allow huge amounts of data to be visualized in public spaces using accessible hardware, Ynnerman et al. have identified a new science communication paradigm that they term exploranation, which combines explanatory and exploratory visualization approaches [5]. While explanatory visualization is applied to communicate scientific findings, exploratory visualization is instead employed by scientists through data analysis tools that allow for interactive exploration of data from measurements and simulations, leading to scientific discovery. Exploranation shares aspects of both approaches to describe interactive visualization of large-scale and/or complex data to communicate science to a non-expert audience. The authors provide three examples of exploranation applications:

- The authors are with the division of Media and Information Technology at the Department of Science and Technology, Linköping University, Sweden. E-mail: konrad.schonborn@liu.se

Manuscript received September 15, 2019 visualization of imaging data captured by computed tomography scanning, intermolecular forces in a biomolecular system based on pre-calculated potential fields, and an astronomy visualization that allows "live" presentation of data from ongoing space missions [5].

Although the exploranation concept was coined only recently, we opine that our previously developed interactive simulation may contain multiple aspects captured by the exploranation term. In turn, this observation initiated a desire to further contemplate and elaborate upon the exploranation concept in an attempt to more rigorously specify the tenets that it may infer. By considering our interactive visualization as an additional potential example of exploranation, we aim to contribute to this discussion.

\section{Accessing Nano through Exploranation}

Our nano simulation, visualization and interaction platform, labelled NanoSim, was developed as part of a science center exhibition about nanoscience and nanotechnology [3]. It was conceptualised and developed for use as an interactive visualization whereby public users could gain access to the otherwise aperceptual properties and physical attributes of the nanoscale. In turn, such interactive experiences were envisaged as a conceptual tool for citizens to scaffold their assessment of the potential risks and benefits of nano for society. We believe that this exhibit represents an important case of science communication for the public from the perspective of exploranation.

\subsection{Public Exhibition Space for Communicating Nano}

We designed and developed NanoSim in the context of an exhibition titled $N A N O$, which premiered during October 2012 at the Norrköping Visualization Center in Sweden. Conceptualising an interactive visualization for the exhibition and then conducting research on learning with the immersive visualization technology was made possible 


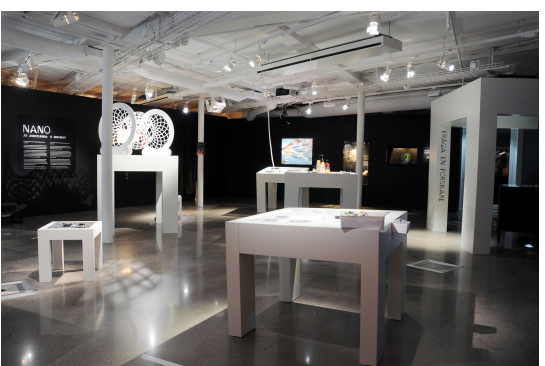

(a) Overview of the NANO exhibition space designed to immerse the visitor in a visual nanoworld that conveys the importance of scale and accompanying nanoprinciples.

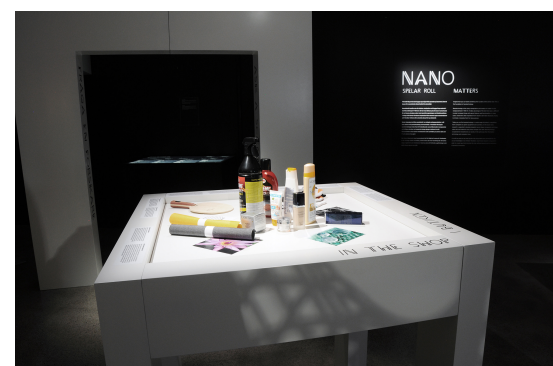

(b) Exhibit demonstrating multiple consumer products that represent human nanotechnological intervention at the nanoscale for rendering their functions at the macroscale.

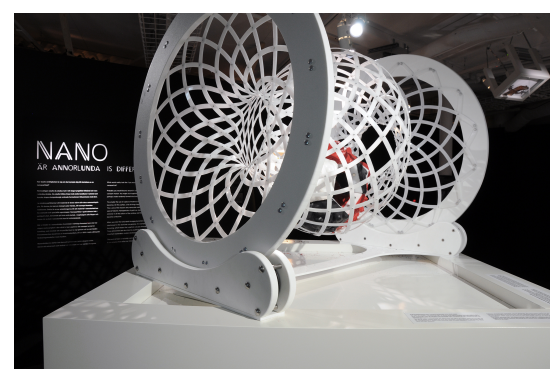

(c) Interactive exhibit on self-assembly. Represented nano-objects containing magnets aggregate via random encounters upon rotation of the "tombola" by visitors.

Fig. 1. Photographs showing examples of various artefacts that were integrated into the NANO exhibition. The differently sized tables in (a) conveyed the importance of scale. The exhibits in (b) and (c) are examples of how multiple scientific principles of nanoscience and nanotechnology were communicated. Photos: Anders Ristenstrand. (C)NVAB, Sweden.

through a research project that we were granted by the Swedish research council.

In mutual collaboration with producers at the center, realisation of the exhibition was in part driven by the NanoForm project, which aimed to develop and strengthen artistic and visual techniques for fostering public access to the nanoscale. The overall idea of NANO was to apply visualization methods to convey the nature and specific properties of the nanoworld, and to highlight the often counterintuitive differences from the tangible macroworld, in order to communicate the impact of nanoscale interactions and nanotechnological intervention for society. Figure 1 provides some examples of the artefacts used in the exhibition. A sense of the overall NANO exhibition space can be gained from Figure 1a. Overall, the exhibition space aimed to immerse the visitor in the represented nanoworld, where various relative size visual cues endeavoured to evoke visitors into contemplating how small nano really is. Some exhibits aimed to make nano tangible by demonstrating currently available consumer products that contain human-manipulated nanoparticles for eliciting their observed macroscale functionalities (e.g. Figure 1b). Other exhibits aimed to contribute to accessing highly abstract yet fundamental nanoscale interactions, such as simulating selfassembly of a virus capsid by physically turning a tombola (Figure 1c).

\subsection{An Interactive Simulation of the Nanoworld}

NanoSim (Figure 2) was integrated into the exhibition with the intention to communicate core nanoscietific concepts through visitors' gestural interaction with virtual nanoparticles (carbon nanotubes). This immersive interaction modelled at the nanoscale serves to provide a basis for developing an understanding necessary to scientifically gauge the perceived promises and dangers of the nanorevolution [4]. Experiencing NanoSim involves users performing various interactions that include deploying a grab gesture to reach into the 3D scene and make contact with virtual nanotubes. Moving, pulling or pushing the nanotubes then provides the basis for engaging with a narrative that purposefully communicates opposing poles of a risk-benefit continuum. The risk scenario represents a potential harmful situation

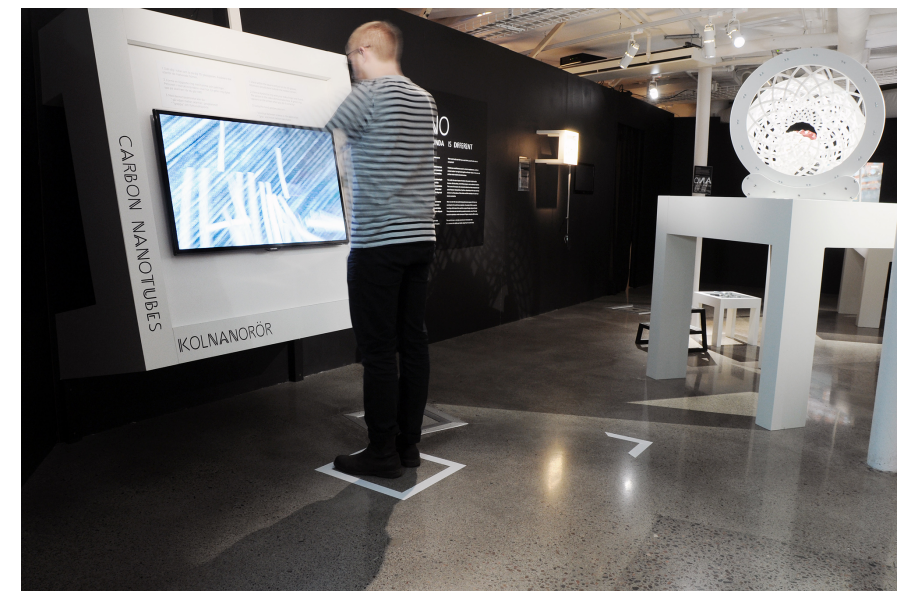

Fig. 2. A user actively interacts with the NanoSim system embedded in the NANO exhibition. The white square on the floor indicates where users should stand for optimal gesture detection and head tracking by the overhead depth camera. The corner angle indicates the boundary of the detection area. Photo: Anders Ristenstrand. (C)NVAB, Sweden.

based on the fact that due to their massive surface area-tovolume and length-to-diameter ratios, mass production of nanotubes could induce a similar hazard to that of asbestos toxicity. The benefit scenario conveys a potential nanotherapeutic application based on the fact that nanotubes can be coated to make them bind specifically to cancer tissue. Given that nanotubes can transfer the absorption of particular infrared frequency as heat, cancer cells can be subsequently eliminated with high precision.

The overall system was arranged as a linear story-telling environment consisting of nine sequential scenes, expressed in conjunction with accompanying text, images, animations and videos. Visitors navigated the scenes by pressing virtual continue or back buttons. Entry into each of the two gesturebased interactive scenarios was described by respective prologue and follow-up scenes.

The NanoSim exhibit was developed as a Virtual Reality (VR) system aimed at providing an immersive and embodied experience. It consisted of a wall mounted 3D TV for displaying stereoscopic content and a Microsoft Kinect depth camera for head tracking and gesture detection. This 


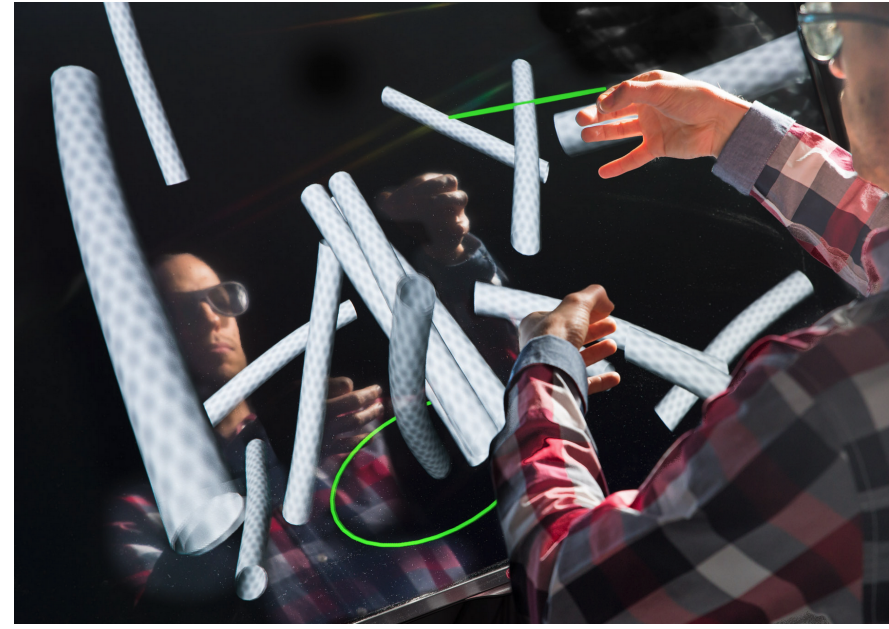

Fig. 3. A user interacts with NanoSim. Forming a ring with the index finger and thumb activates a "grab" event in the corresponding position in the virtual space, indicated by a green ring. The "grab" gesture allows the user to clasp and move the virtually attached nanotube. (C)Thor Balkhed.

setup allowed visitors to interact with the exhibit without physically handling any physical objects apart from the 3D glasses required for a correct stereoscopic view.

Users interacted with the exhibit through gestures that the depth camera monitored through custom algorithms developed to allow placement of the camera above the display and user rather than in front. Three features were extracted from the depth image:

1) a large object closest to the camera, interpreted as the head of the visitor,

2) a small object furthest down in the image, closest to the display, interpreted as a finger used to activate virtual buttons, and

3) circles of a pre-defined size and shape, interpreted as thumb and index finger contacts performed by visitors during deployment of a grab gesture.

The implementation resulted in effective head tracking. Visitors perceived the nanotubes as protruding from the screen and they often moved around to view the nanotubes from other angles or to simply experience the motion parallax [6]. They could also simultaneously grasp nanotubes with both hands (Figure 3).

The visualization of nanoparticle behaviour was based on a real-time simulation running in the background. To run at an interactive rate, it was not possible to simulate interaction between individual atoms. The simulation was instead based on a physical engine that could detect collisions between nanotubes modelled as tube-formed interlinked segments. A simple model of the interaction between molecules, based on the Lennard-Jones potential for interaction between neutrally charged molecules, was used to simulate intra- and inter-tube interaction. In addition, to simulate Brownian motion, random movement was included by adding random impulses to the movement of each tube segment.

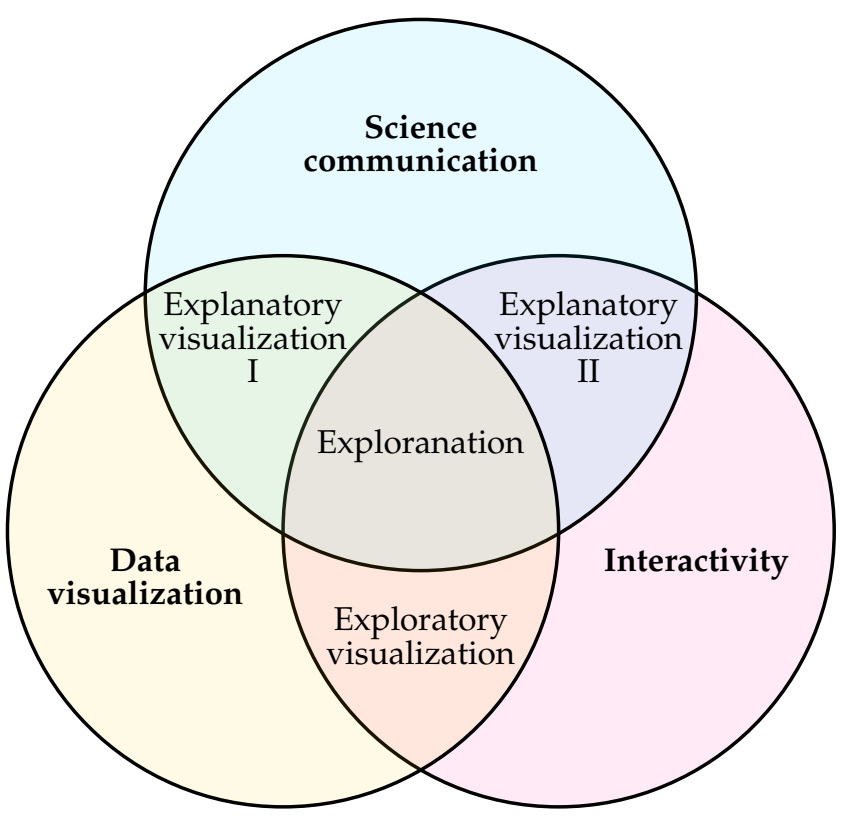

Fig. 4. Venn diagram illustrating how exploranation emerges from the simultaneous engagement of the three overarching components of Science communication, Data visualization and Interactivity. Combining two components yields exploratory visualization, or one of two different types of explanatory visualization, respectively.

\subsection{NanoSim as an Exploranation Environment}

Our interpretation of exploranation is that it is an expression of the merging of three overarching components, namely Science communication, Data visualization and Interactivity, as shown in Figure 4. According to this interpretation, exploranation is seen as a unique concept that can be distinguished from other approaches to visualizations. In this regard, we note that the Venn diagram in Figure 4 allows us to distinguish between two different forms of explanatory approaches to visualization that convey scientific concepts through distinct combinations of these components. First, we can see how the visualization of large scale and complex scientific data can support learning of scientific concepts, such as an animation that conveys the nanoworld by zooming into image data of progressively higher resolution. This is denoted by Explanatory visualization I in Figure 4. Second, scientific concepts can be demonstrated through interactive visualizations of simplistic models. For example, the concept of molecular polarity can be investigated by interactively altering the electronegativities of two atoms in a simple model. We denote this as Explanatory visualization II in Figure 4. In a similar fashion, as inferred in Figure 4, scientific data visualization that is performed as part of the research process rather than for the purpose of communication becomes Exploratory visualization when an interactive component is added, such as using a tool to interactively analyze a confocal microscopy dataset.

In representing the combination of all three components in Figure 4, it is to be expected that an exploranation application exhibits features of explanatory as well as exploratory visualization. Here, NanoSim clearly incorporates techniques and conventions from explanatory visualization approaches to communicate nanoscience. For instance, established visual conventions for visualizing molecules are 
employed in the "stick" representations of the nanotubes that visitors can interact with in the simulation (Figure 3), but also in the contextual information provided to visitors before entering the interactive scenes. In addition, the follow-up scenes included images at different levels of scale obtained from research publications to illustrate events related to nanotube exposure, to reflect the danger of inhaling nanotubes in the risk scenario and the therapeutic action of modified nanotubes in the benefit scenario, respectively.

In addition to explanatory visualization, NanoSim also employs techniques from exploratory visualization of nanoscientific phenomena by employing interactive simulation, a pivotal research tool in the molecular sciences. Traditional explanatory approaches would typically use animations of nanoparticles to account for molecular movements and interactions, animations with dynamics that have been designed in advance to convey a desired outcome, and which do not allow for meaningful user interaction. In contrast, although the simulation produced by NanoSim was not part of a nanoscience research project, the dynamic properties of NanoSim are based on techniques and principles that are described in the scientific literature for accurately simulating molecular interaction, albeit in more elaborated forms.

A "coarse-grained" approach was adopted to allow the simulation to run at an interactive rate. This is a common solution for exploring the interaction between large molecules where it is often not possible to simulate interaction between individual atoms [7]. Here, molecular systems with a large number of atoms are approximated through a smaller set of particles that interact via empirical force fields (such as the Lennard-Jones potential). Similarly, the random movement of molecules arising from interactions with surrounding solvent molecules (Brownian dynamics) is typically simplified to minimise the number of molecules included in the simulation [8].

Finally, allowing visitors to interactively interfere with the running simulation (computational steering) is an established approach that can make complicated simulations more efficient by allowing domain experts to focus the simulation on desired states of the investigated system [9].

\subsection{Considering Design Principles of Exploranation}

Ynnerman et al. [5] have proposed four design principles for exploranation applications. Although these were not yet conceptualised during the development of NanoSim, it is compelling to consider points of alignment and divergence with respect to the exploranation paradigm. The design principle "Interleaving Explorative Microenvironments and Signposted Narratives" proposes a structure where users are invited to explore selected parts of data with annotations or other explanations in exploratory sessions that are subsumed into an overall narrative structure. This principle is supported to a degree in NanoSim, in that episodes of interactive exploration are preceded and followed by information screens with information to explain the context and implications of the simulations. However, it is possible that visitors could have benefited from some form of support for their interpretation of the complex scientific content. For example, the contextual information could have been inte- grated in the interactive episodes to direct users' attention to events that occur during the simulation.

The conflicting requirements implied by the design principle "Constraining Explorative Interactions while Leveraging Pliability" were explicitly engaged with while developing NanoSim. The principle emphasizes the need to balance exploratory freedom with constraints to avoid experiences that are disappointing or difficult to understand for users. In NanoSim, the resulting system was made flexible in the sense that users could interact freely with all simulated objects in each of the two interactive scenes. At the same time, interaction was constrained in that the actions that they could perform were limited to "grabbing" and "moving" nanotubes. Informal observations indicate that some users were unsure of what they were expected to do. Hence, the open-ended nature of the interaction may have created uncertainty among users.

The design principle "Foregrounding the Topic through Perceptual Layering" is difficult to apply to the NanoSim case. The principle refers to designing the interface and additional information in ways that does not interfere with the explored phenomena, which should always be the main focus. As shown in Figure 3, the exploratory interface of NanoSim only contains the simulated objects, and therefore there was no perceptual layering in the same sense as described by Ynnerman et al. [5]. However, although this design served to maintain focus on the topic at hand, it is possible that users could have benefited from some form of support for their interpretation of the simulation environment. For example, information signs that explain what the different objects represented could have been included.

The design principle "Supporting Performative Interaction" refers to the potential of users taking on different roles, where a user may "perform" with the exploranatory system in front of an audience of bystanders. This principle is accounted for to some degree in NanoSim. It is possible for quite a large group of visitors to share the same view of the screen in a way that would not be possible if the immersive system was based on a head mounted display. However, they all require 3D glasses to obtain a stereoscopic view of the scene. In addition, the perspective would only be completely correct for one person.

In hindsight, the design principles discussed above could have provided valuable guidance in designing NanoSim. Most importantly, the user experience could have been improved by incorporating annotations and other types of information in the interactive sessions. In turn, this could have reduced the uncertainty about what users were expected to do that arose from the open-ended nature of interacting with the continuously running interactive simulation. Moreover, the potential for performative interaction might be better exploited using emerging display technology that does not require specialized viewing equipment.

\subsection{Exploranative Learning Events with NanoSim}

In previous work [6] we have shown that users of the NanoSim system have an immersive experience and that the system stimulates interaction with and learning about nanophenomena. By engaging the three components of exploranation, we have observed how the "confluence of 
exploration and explanation" [5] is directly observable from users' interaction with NanoSim. To demonstrate this, we have selected three examples of observations of public visitors' interaction with NanoSim. The data were drawn from video-recordings of 40 public citizens' interaction with the system, during open-ended think-aloud interviews. We believe that these examples are moments where exploration via direct interaction with scientific visualized data provides a pathway for successful explanation in the form of communication of an intended scientific message (cf. visualizations for "exploratory explanation" [10]).

In the first example, the interviewer (I) engages in a dialogue with a user (U1) regarding any differences between observing and being able to manipulate the rendered carbon nanotubes. The following exchange ensues:

I: What is the difference between observing and being able to move the tubes? [...] How does it differ, when you are able to move the objects around?

U1: They want to have a big surface [in contact]... It's more obvious when you are moving it [the nanotube]... Yes, I got that knowledge now when I can actually move them [...] To get them off the surface I really have to pull them away...

I: ...Does that help you see something that you didn't see before?

U1: ... I'm more convinced at their "ability" to connect to surfaces... because when I grab them, I really have to grab them to get them off the [cell] wall.

The excerpt suggests that the opportunity to actually move and interact with the visualized nanotubes allows the user to acquire the scientific message that surface area contact between nano objects is a pivotal factor in nanoscale forces. Herein, the fact that the user can interactively explore the nano dynamics by actively moving and pulling nanoobjects from one-another serves to explain the fundamental nanoscientific concept that forces between nanoscale objects are stronger when the surface contact area between them is larger.

In a similar situation to that above, a second example takes the form of I asking a user (U2) to compare only visually observing the behaviour of the modelled nanotubes in solution versus interacting with them, which leads to the following response:

I: ... now that you are interacting with the tubes, can you provide any differences between only observing and interacting with the tubes in terms of the knowledge that you were getting from the simulation?

U2: ...When you can't "touch" them, and they're just hanging [free in solution]... you don't understand how they are moving... [...] But now I see... I see that they are all together [aggregated] might just be a random thing that happens... Because, when you can't move them, you just think that they all "want" to get into a group together.

The exploratory aspect offered by being able to grab and move the virtual nanotubes, can pave the way for a user to discern the explanation that forces between nanoscale objects in solution arise when the objects are near each other by chance. Moreover, this sets the scene for a preliminary conceptualization of the counter-intuitive process of selfassembly, where nanoscopic objects spontaneously aggregate through random encounters.

In a third and final example, a user (U3) interacts with modified nanotubes that bind to receptors expressed on the surface of a cancer cell, whereupon I induces the following exchange:

U3: Let me grab one [modified nanotube] like this...

I: What defines the right or wrong "socket" (U3 earlier referred to a receptor as a socket) [for binding]?

U3: Colour and shape... usually the types of atoms.

I: Anything more about their attachment?

U3: They don't stick for a long time really [...]

I: What will determine whether there is going to be a connection made between a socket and a yellow structure [binding molecules are yellow halfspheres on the modified tubes], based on where they are at a particular moment in time... is there anything that "decides", so to say, whether there will be an attachment or not?

U3: Apart from the [type of] "socket", it [modified nanotube] has to come close enough.

I: How does that happen?

U3: By luck... Not all of them [modified nanotubes] stick, and not all of them go to the right place... it [a dosage of tubes] doesn't all go there straight away.

Consideration of the excerpt above demonstrates how the user's exploration of the binding between a cancer cell receptor and molecule by grabbing, pulling and placing of a modified nanotube provides a gateway for the explanation that complementary molecular shapes underlie selective binding at the nanoscale (molecular recognition). Furthermore, this exploratory opportunity also allows the user to note that modified nanotubes that have attached to their specific target do not remain permanently bound. Moreover, the binding event is then conceptualised by the user as only occurring when the objects are near each other by chance. Together, these experiences provide the basis for the core realisation that the targeted drug delivery metaphor does not imply that medical substances traverse directly towards a "target" after being introduced into the body.

Overall, a striking observation in the three presented examples is that the science communication induced by users' interaction with the data is manifested in an everyday language, divorced from domain-specific scientific terminology. In these cases, public users communicate their explanations of otherwise abstract and complex nanoscientific concepts in terms of exploratory experiences that emanate directly from interacting with the visualized data themselves. We suggest that such episodes, where the three components depicted in Figure 4 are simultaneously activated, could be seen as distinct exploranative learning events. 


\section{Practical Considerations}

Our retrospective analysis of the NanoSim exhibit from an exploranation perspective has provided multiple insights. For example, we find that challenges encountered in developing NanoSim reflect all three components of exploranation (Figure 4). In the following, we reflect on challenges encountered in developing the exhibit and its potential as a learning tool in the public exhibition.

\subsection{Science communication}

Developing an exhibit in the context of an exhibition (see Section 2.1) places restrictions and contextual demands for the exhibit to fit in harmony with the rest of the exhibition. The exhibit must fulfil a useful role in the overarching narrative structure of the exhibition as a whole, but the contextual demands also include the physical conditions offered by the exhibition space, such as lighting and exhibit placement relative to other exhibits.

An exhibit must be suitable for the intended audience of the overall exhibition. This involves accounting for the expected level of scientific knowledge among visitors. While science communication for a general public requires simplifications, there is always a risk that the science is presented in misleading ways that may create or reinforce incorrect conceptions of structures and processes in nature. As discussed in Section 2.4, integrating explanatory information into the interactive sessions may be one avenue for supporting users' interpretation of the visualized information.

Overall, communicating advanced and abstract nanoscience and nanotechnology in an accessible way to a heterogeneous audience was a challenge not only for designing NanoSim but for the entire exhibition. While the conceptual challenges in terms of science content differs between applications, understanding and managing visitors' pre-knowledge is clearly a crucial component of developing successful exploranation applications.

\subsection{Data Visualization}

Communication of a science-oriented narrative also requires consideration of the mapping between the interactive visualization and the structure of the underlying scientific concepts. For example, we employed a "rubber band" analogy to emphasize the forces that users apply to the simulated objects. This consisted of a graphically generated orange line between the green ring that formed after a grab gesture was performed and the nanotube that was being engaged through the interaction. As users moved their hands, the "rubber band" would increase or decrease in length to reflect an increased or decreased external force being applied to the simulated object.

While serving to focus users on the interaction at hand from a perceptual point of view, applying a macroscopic rubber band analogy for the visual feedback that follows users' interaction with the nano scale scenario is not without its risks. In some rare cases visitors appeared to interpret the interactive engagement with the user interface as a genuine representation of how nanoscopic manipulation of matter might be performed. Rather than viewing the exhibit as an interactive data visualization regarding the physical behaviour of nanotechnological artefacts, they viewed it as a representation of an existing nanotechnological workflow. Thus, the connection between the interactive and visual features of a visualization may not always be immediately clear to users.

Another important aspect of adapting the data visualization to the audience was establishing the appropriate level of exploratory freedom that visitors could handle while still providing a meaningful experience. For example, it would in principle have been possible to allow users to manipulate simulation parameters, or change visual representations, but this would likely have made the experience less accessible for many visitors.

\subsection{Interactivity}

As pointed out by Ynnerman et al. [5], the interaction interface must be sufficiently physically robust to avoid breakdowns following public engagement with the exhibit, which can sometimes be quite physical. We confronted this challenge by designing an interaction interface that allowed 3D interaction without actually touching any sensitive equipment. While this design decision served to reduce greasing and wear, the lack of a physical interface precluded the use of, for example, haptic equipment, which could have provided a visuohaptic experience for visitors (cf."MolDock" [5]).

In addition to physical hardiness, the software must also be stable. Given that the exploratory visualization systems used in scientific research are often at an early stage of development, there is a limit to how close to the current scientific developments exploranation applications can be.

Furthermore, unsupervised engagement in immersive exploration and interaction requires an intuitive interface which is easy to manage for new users [5]. This turned out to be a difficult challenge in designing the NanoSim exhibit. Despite the embodied and intuitive basis for engaging with your surroundings using your hands, the requirement of a machine-readable signal for the action of "grabbing" objects posed a pedagogical dilemma.

Forming a circle using the thumb and index finger to "pinch" nanotubes came quite natural to most users. However, problems arose because users quickly forgot (or never realized) that the system was only able to detect wellformed circles in the right orientation relative to the depth camera. Thus, users would sometimes be found pinching in the air to form more or less round circles oriented in angles that the depth camera could not detect, leading to frustration when the action was not followed by the anticipated visual feedback from the simulation. Hence, the pinch gesture adopted for user interaction may arguably have been too intuitive given the limited capacity of the system to detect pinch gestures that did not conform to the technical prerequisite for interaction. Combining an intuitive basic gesture with non-intuitive restrictions on the performance of the gesture is clearly problematic.

In developing visualizations that form part of an exhibition, the interactive affordances offered by other exhibits should also be considered. For example, can the same types of interactive gestures and actions be used in multiple exhibits? Minimizing the number of interactive behaviours 
that need to be mastered could serve to make the exhibition as a whole more accessible for users. In this regard, the relative lack of conventionalized gesture interaction in 3D environments compared to $2 \mathrm{D}$ touch interfaces presents a challenge for developers of exploranation applications.

\subsection{Potential for Technological Refinements}

Any development will be limited by the existing technology that is available when the system is built. The first commodity head mounted display (HMD), or VR headset, was released almost one year after the opening of the NANO exhibition. Thus, our display system for immersive 3D visualization and interaction had little choice but to employ an alternative Virtual Reality design principle, in our case a room mounted display (RMD). However, even today we would probably still employ an RMD if given the opportunity to deploy our system in a new guise. To date, headsets are somewhat cumbersome to use, and require supervision to adjust. This introduces an unwanted threshold that also slows down hand-over between visitors. Furthermore, current headsets shut out the real world by completely covering one's vision, which can be both mentally unpleasant and potentially dangerous. In contrast, we felt that we managed to build a display system for NanoSim that invited natural and spontaneous visitor interaction.

Recent technology could provide several potential improvements to use in future deployments of immersive exploranation exhibits. For example, we introduced an unwanted threshold by merely requiring that visitors should realize that they need stereoscopic glasses for the 3D TV and that they should actually retrieve them for use. This somewhat reduced the effortless interaction with our system. Thus, we welcome the increasing quality of autostereoscopic displays and hope that these will soon reach a level where they are also suitable for high quality Virtual Reality scenarios. Recent developments in camera-based face tracking can also be used to more accurately adjust the view, for an even more realistic and thereby exciting experience. Such an approach could more effectively control which visitor to adjust the view for, and thereby preventing spectators from inadvertently "stealing the perspective" from the current user of the system, or even to select an approximate view between two users.

Another partly technical issue is the observed problem concerning the grab gesture, requiring visitors to position their hand so that the formed circle was clearly visible from above where the depth camera was mounted. Thus, while detecting circles for interaction with the virtual objects in the application worked well, in hindsight it would have been preferable if the algorithm could track individual fingers and detect a natural grasp action. This was not feasible with the technology available at the time the system was built.

Both hardware and algorithms for depth camera tracking have improved and it is now possible to track individual fingers and detect dexterous actions with increasing accuracy and robustness. However, our experience with the NanoSim exhibit emphasizes the importance of choosing a style of interaction which is both accurate and robust; users get easily distracted and frustrated by any inconsistent reactions to their actions. In this regard, the designer will have to establish a trade-off between using more abstract gestures, that are more easily and accurately detected through camerabased tracking, and hand actions that may be more natural and intuitive but more difficult to detect.

Finally, even though it is true that visualization and interaction methodology has advanced to a mature stage [5], the computational demands of interactive simulations still limits the size of systems that can be included. We can thus assume that as computational power evolves, so will the potential for more advanced visual representations as well as simulation of larger systems with higher resolution and frequencies in future exploranation applications.

\section{Developing the Exploranation Paradigm}

Conceptualizing exploranation in terms of the Venn diagram in Figure 4 emphasizes the integration of the three components. It provides a helpful heuristic for distinguishing between exploranation approaches on one hand, and other kinds of approaches to visualization and science communication on the other hand. Furthermore, it provides a framework for structuring design and analysis considerations in relation to three important aspects of any exploranation application. We believe that such a framework is helpful in disentangling and focusing issues that are otherwise obscured by the overall complexity of exploranation applications. For example, in composing this article, we have reflected on different types of data that could be included in exploranation applications.

We note that two important distinctions are whether the data is static or dynamic, and whether it originates from measurement devices or constitutes the output from simulations based on scientific models. These distinctions indicate four different ways that the Data visualization component can be realized: static measurement data (e.g. visualization of scanned medical data); static simulated data (e.g. visualization of pre-calculated molecular force fields); dynamic measurement data (e.g. visualization of data sent from space probes); and dynamic simulated data (e.g. visualization of data from computational steering of a simulation). In Figure 5, the examples from Ynnerman et al. [5] are placed within this matrix together with NanoSim.

In this manner, analysis of existing or planned exploranation applications could be done to establish relevant distinctions within each of the Data visualization, Science communication and Interactivity components, respectively, to further map out the conceptual landscape on which the exploranation paradigm may operate. We believe that research programmes aiming to further explore exploranation should consider establishing such a framework, which could in turn provide a potential baseline for discussing and comparing exploranation studies.

\section{Conclusion}

We are of the view that computed dynamic data, as exemplified through the NanoSim exhibit, can further complement the concept of exploranation. It is, however, possible that the different nature of the data places different demands on the design and contextualization of the narrative structure. One obvious possibility is that exploring static structures may differ from exploring dynamic processes. In addition, 


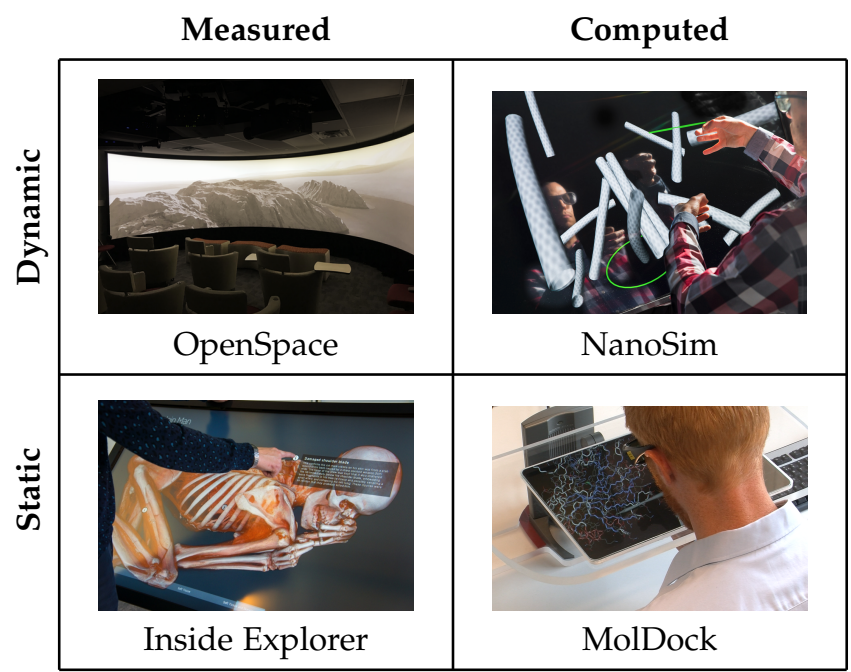

Fig. 5. A matrix for relating current examples of exploranation applications to the properties of the underlying data in terms of whether it is Static or Dynamic, and whether it is produced via Measurements or Computational methods. (C) OpenSpace Project, (CRISE Interactive, (C)Thor Balkhed.

we note that all of the exploranation examples that have been put forward so far (Figure 5) concern data which are structured in three spatial dimensions. One open question is whether the observed trend towards a confluence of exploration and explanation is also relevant for scientific domains where data are more abstract and multidimensional (e.g. bioinformatics), or in fields that are not physical science (e.g. history). Thus, further development of the theoretical principles and scope of the constructs of exploranation are exciting points of departure that await further probing from our community.

We also believe that together with recent technology, the inviting openness of the immersive and interactive room mounted display system fits well into the future of exploranation in public spaces, making immersive exhibits a viable alternative to increasingly popular touch screens and touch tables. In particular, through the application of Virtual Reality technologies, this approach provides the spatial understanding of three dimensional structures and concepts, and scales, while still affording direct hand interaction with the intended visualization content.

\section{ACKNOWLEDGMENTS}

The Swedish Research Council (Vetenskapsrådet, grant 2011-5569), Norrköping Visualization Center C (NVAB), and the NanoForm project organization supported this work.

\section{References}

[1] H. A. Yacoubian, "Scientific literacy for democratic decision-making," International Journal of Science Education, vol. 40, no. 3, pp. 308-327, 2018. [Online]. Available: https://doi.org/10.1080/09500693.2017.1420266

[2] J. K. Gilbert and H.-S. Lin, "How might adults learn about new science and technology? The case of nanoscience and nanotechnology," International Journal of Science Education, Part B, vol. 3, no. 3, pp. 267-292, 2013.

[3] K. L. Palmerius, G. Höst, and K. Schönborn, “An interactive and multi-sensory learning environment for nano education," Lecture Notes in Computer Science, vol. 7468, pp. 81-90, August 2012.
[4] K. Schönborn, G. Höst, and K. L. Palmerius, "Nano education with interactive visualization," Nano Today, vol. 11, no. 5, pp. 543-546, 2016.

[5] A. Ynnerman, J. Löwgren, and L. Tibell, "Exploranation: A new science communication paradigm," IEEE Computer Graphics and Applications, vol. 38, no. 3, pp. 13-20, 2018.

[6] K. J. Schönborn, G. E. Höst, K. E. L. Palmerius, and J. Flint, "Development of an interactive immersion environment for engendering understanding about nanotechnology: Concept, construction, and implementation," International Journal of Virtual and Personal Learning Environments, vol. 5, no. 2, pp. 40-56, 2014. [Online]. Available: https://doi.org/10.4018/ijvple.2014040104

[7] J. Zhao, J.-W. Jiang, L. Wang, W. Guo, and T. Rabczuk, "Coarsegrained potentials of single-walled carbon nanotubes," Journal of the Mechanics and Physics of Solids, vol. 71, p. 197218, 2014.

[8] G. W. Slater, C. Holm, M. V. Chubynsky, H. W. de Haan, A. Dub, K. Grass, O. A. Hickey, C. Kingsburry, D. Sean, T. N. Shendruk, and L. Zhan, "Modeling the separation of macromolecules: A review of current computer simulation methods," ELECTROPHORESIS, vol. 30, no. 5, p. 792818, 2009. [Online]. Available: https://onlinelibrary.wiley.com/doi/abs/10.1002/elps.200800673

[9] M. Dreher, M. Piuzzi, A. Turki, M. Chavent, M. Baaden, N. Frey, S. Limet, B. Raffin, and S. Robert, "Interactive molecular dynamics: Scaling up to large systems," Procedia Computer Science, vol. 18, p. 2029, 2013, 2013 International Conference on Computational Science.

[10] N. Iliinsky and J. Steele, Designing data visualizations: Representing informational Relationships. "O'Reilly Media, Inc.", 2011. 